\title{
Galactic Evolution of Beryllium and Oxygen
}

\author{
Garik Israelian ${ }^{1,2}$, Ramón J. García López ${ }^{1,2}$ and Rafael Rebolo ${ }^{1,3}$ \\ 1.- Instituto de Astrofísica de Canarias, E-38200 La Laguna, \\ Tenerife, Spain \\ 2.- Departamento de Astrofísica, Universidad de La Laguna, Av. \\ Astrofísico Francisco Sánchez s/n, E-38071 La Laguna, Tenerife, Spain \\ 3.- Consejo Superior de Investigaciones Científicas, Spain
}

\begin{abstract}
We discuss the early evolution of beryllium and oxygen in our Galaxy by comparing abundances of these elements for halo and disk metal-poor stars. Both, $\mathrm{O}$ and $\mathrm{Be}$ rise as we go progressively to more metal-rich stars, showing a slope $0.41 \pm 0.09([\mathrm{Be} / \mathrm{O}]$ vs $[\mathrm{Fe} / \mathrm{H}])$ for stars with $[\mathrm{Fe} / \mathrm{H}] \leq-1$. This relationship provides an observational constraint to the actually proposed Galactic Cosmic Ray theories.
\end{abstract}

\section{Introduction}

First attempts to measure beryllium abundances in metal-poor stars by Molaro \& Beckman (1984) and Molaro, Beckman \& Castelli (1984) demonstrated that stars in the early Galaxy formed with much lower Be abundances than in the present epoch. First detection of Be in metal-poor stars was achieved by Rebolo et al. (1988) and further studies by S. Ryan, G. Gilmore, A. Boesgaard, P. Molaro, R. J. García López and their respective collaborators revealed a clear linear correlation with iron.

Accelerated protons and $\alpha$-particles in cosmic rays interact with ambient CNO in ISM and create Be. According to the standard Galactic Cosmic Ray (GCR) theory, these interactions in the general ISM should have given a quadratic relation between $\mathrm{Be}$ and $\mathrm{O}$. Alternatively, spallation of cosmic ray CNO nuclei accelerated out of freshly processed material could account for the primary character of the observed early galactic evolution of Be. Another production site is the collective acceleration by SN shocks of ejecta-enriched matter in the interiors of superbubbles. In these two cases, the evolution of $\mathrm{Be}$ should reflect the production of CNO from massive stars. Oxygen is mostly produced by Type II SNe while iron is produced in both, Type II and in Type Ia SNe. The fact that Type Ia SNe have longer lifetime progenitors has been commonly used to argue that oxygen must be overabundant in very old stars. Observational evidence for high $[\mathrm{O} / \mathrm{Fe}]$ ratios in many metal-poor stars has been reported over the last two decades.

Based on the study of [O I] lines at 6300 and $6363 \AA$ in evolved stars (though the second line at $6363 \AA$ is not visible in very metal-poor stars and the analysis is based only on one line), several authors have found that $[\mathrm{O} / \mathrm{Fe}]=0.3-0.4 \mathrm{dex}$ at $[\mathrm{Fe} / \mathrm{H}]<-1$ and is constant until $[\mathrm{Fe} / \mathrm{H}] \sim-3$ (e.g. Barbuy 1988 and Kraft et 
al. 1992). In contrast with this result, oxygen abundances derived in unevolved stars using the O I IR triplet at $7774 \AA$ (Abia \& Rebolo 1989; Tomkin et al. 1992; King \& Boesgaard 1995; and Cavallo, Pilachowski, \& Rebolo 1997) point towards linearly increasing $[\mathrm{O} / \mathrm{Fe}]$ values with decreasing $[\mathrm{Fe} / \mathrm{H}]$ and reaching a ratio $\sim 1$ for stars with $[\mathrm{Fe} / \mathrm{H}] \sim-3$. This may suggest a higher production of oxygen during the early Galaxy.

We discuss in this paper the comparison of these abundances with those derived from $\mathrm{OH}$ lines located in the near-UV part of the spectra of metal-poor stars, and their relation with beryllium abundances consistently derived from the same spectra.

\section{Observations and Analyses}

The observations were carried out in different runs using the UES $(R=\lambda / \Delta \lambda \sim$ 50000 ) of the 4.2-m WHT at the Observatorio del Roque de los Muchachos (La Palma), and the UCLES $(R \sim 60000)$ of the 3.9-m AAT. The spectral region observed spanned typically from 3080 to $3300 \AA$, where several $\mathrm{OH}$ lines and the Be II doublet at $3131 \AA$ are located. Details of the abundance analyses can be found in García López, Severino, \& Gomez (1995) and Israelian, García López, \& Rebolo (1998) for beryllium and oxygen, respectively. The stellar parameters play an important role in the abundance determinations from near-UV lines. Effective temperatures ( $\left.T_{\text {eff }}\right)$ for our stars were estimated using the Alonso et al. (1996) calibrations versus $V-K$ and $b-y$ colors, which were derived by applying the infrared flux method (IRFM), and cover a wide range of spectral types and metal content. These temperatures were used to compute synthetic spectra around the Be II doublet and slightly modified, within the error bars provided by the calibrations, until obtaining a good reproduction of this region in the observed spectra. Metallicities were adopted from literature values obtained from high resolution spectra. Adopted gravities, derived using the accurate parallaxes measured by Hipparcos (ESA 1997), are larger by $0.28 \mathrm{dex}$ in average than the values adopted by Israelian et al. (1998). This implies a mean small reduction of 0.09 dex in the oxygen abundances inferred from the $\mathrm{OH}$ lines with respect to that work, which does not affect significantly their original results.

Beryllium abundances reported here were obtained from the Be II resonance doublet located at 3130.421 and $3131.065 \AA$. The first line, which is also the strongest one, is severely blended with atomic and molecular lines of other species, and the abundance determination usually relies only on the other line, more isolated and weaker. The abundances used in this work are those presented in García López (1999).

\section{Oxygen}

Israelian et al. (1998) presented new oxygen abundances derived from nearUV OH lines (which form in the same layers of the atmosphere as [O I]) for 24 metal-poor stars. They have concluded that the $[\mathrm{O} / \mathrm{Fe}]$ ratio of metal-poor stars increases from 0.6 to 1 between $[\mathrm{Fe} / \mathrm{H}]=-1.5$ and -3 , with a slope of $-0.31 \pm 0.11$. Contrary to the previously accepted picture (see e.g. Bessell, Sutherland, \& 
Ruan 1991, who used older model atmospheres with a coarser treatment of the opacities in the UV), these new oxygen abundances derived from low-excitation $\mathrm{OH}$ lines, agreed well with those derived from high-excitation lines of the O I IR triplet at $7774 \AA$. The comparison with oxygen abundances derived using O I data from Tomkin et al. (1992) showed a mean difference of $0.00 \pm 0.11 \mathrm{dex}$ for the stars in common. Boesgaard et al. (1999a) made a similar analysis of several metal-poor stars using a different set of $\mathrm{OH}$ lines. They found a very good agreement with the results obtained by Israelian et al. (1998), and basically the same dependence of $[\mathrm{O} / \mathrm{Fe}]$ versus metallicity. This is clearly seen in the upper panel of Figure 1.

The UV "missing opacity" problem discussed by Balachandran \& Bell (1998), which could affect both oxygen and beryllium abundance determinations from these lines, has been studied recently by Allende Prieto \& Lambert (2000). These authors have found a good agreement between $T_{\text {eff }} \mathrm{S}$ obtained from the IRFM and from the near-UV continuum for stars with $4000 \leq T_{\text {eff }} \leq 6000 \mathrm{~K}$ when accurate Hipparcos gravities are used. This also agrees with our good reproduction of the near-UV spectral region using the IRFM temperatures. This result indicates that the model atmospheres used provide an adequate description of the near-UV continuum forming region. In any case, even if a not well understood opacity problem would exist as described by Balachandran \& Bell, it would have a minor effect on the $\mathrm{OH}$ results since most of the stars in the samples of Israelian et al. and Boesgaard et al. are hotter than the Sun and very metal-poor. The corrections to oxygen abundances for individual stars would be lower than 0.15 dex, not changing significantly the $[\mathrm{O} / \mathrm{Fe}]$ vs. $[\mathrm{Fe} / \mathrm{H}]$ trend.

A new non-LTE analysis of the O I IR triplet for a sample of 38 metal-poor stars performed by Mishenina et al. (2000) gives consistent results with those of Abia \& Rebolo (1989), Tomkin et al. (1992) and Kiselman (1993), and indicates that the mean value of the non-LTE correction in unevolved metal-poor stars is typically $0.1-0.2$ dex. These authors confirmed the $[\mathrm{O} / \mathrm{Fe}]$ vs $[\mathrm{Fe} / \mathrm{H}]$ trend discussed by Israelian et al. (1998) and Boesgaard et al. (1999a) from the $\mathrm{OH}$ lines, without finding any trend of oxygen abundances with $T_{\text {eff }}$ or $\log g$. It is also worthwhile to mention that the O I IR triplet is not affected by $3 \mathrm{D}$ effects, convection and small-scale inhomogeneities in the stellar atmosphere (Asplund et al. 1999). In addition, oxygen abundances derived form this triplet are not significantly affected by chromospheric activity either. The central panel of Fig. 1 shows a compilation of oxygen abundances derived using the IR triplet. The larger scatter observed as compared with the measurements based on $\mathrm{OH}$ lines can be associated with the different scales of stellar parameters ( $T_{\text {eff }}$, gravities, and metallicities) adopted by the authors of each set of stars, and to the fact that some measurements have not been corrected for non-LTE effects. Very recently, Carretta, Gratton, \& Sneden (2000) performed an independent analysis of 32 metal-poor stars hotter than $4600 \mathrm{~K}$ using the IR triplet, and provide LTE and non-LTE oxygen abundances which are significantly lower than those found in previous works. A preliminary attempt to understand the reasons for this discrepancy can be done by looking in detail into their most metal-poor star $\left(\mathrm{BD}+3^{\circ} 740,[\mathrm{Fe} / \mathrm{H}]=-2.66\right)$ where a surprisingly low oxygen abundance $[\mathrm{O} / \mathrm{Fe}]=0.38$ is claimed. A recent study of stellar parameters based on the non-LTE analysis of iron lines (Thévenin \& Idiart 1999), gives a lower effective temperature (by $140 \mathrm{~K}$ ) and a higher gravity (by 0.3 dex) than the 


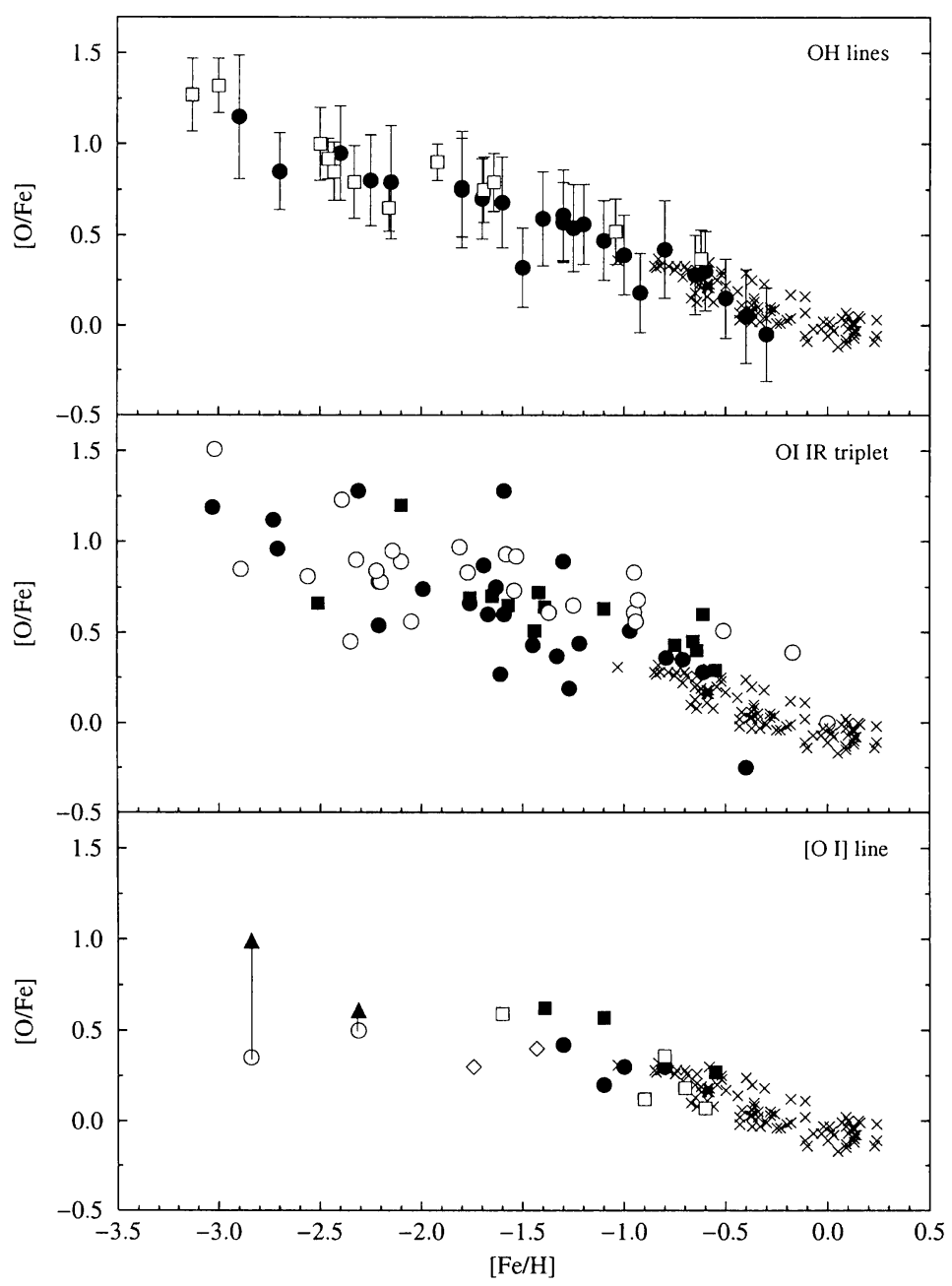

Figure 1. $[\mathrm{O} / \mathrm{Fe}]$ vs. $[\mathrm{Fe} / \mathrm{H}]$ for unevolved stars. Abundances from $\mathrm{OH}$ lines were derived by Israelian et al. (1998; filled circles) and Boesgaard et al. (1999a; open squares, corrected to the scale of stellar parameters adopted by Israelian et al.). Abundances from the IR triplet were derived in NLTE by Mishenina et al. (2000; filled circles), Cavallo et al. (1997; filled squares, corrected for NLTE effects by Mishenina et al.), and in LTE by Boesgaard et al. (1999; open circles). Finally, abundances from the [O I] line come from Spiesman \& Wallerstein (1991; open diamonds), Spite \& Spite (1991; open squares), Israelian et al. (1998; filled circles), Mishenina et al. (2000; filled squares), and Fulbright \& Kraft (1999; open circles). Filled triangles indicate the change in abundances associated with the change in gravity according to the Hipparcos parallaxes for the two stars studied by Fulbright \& Kraft. The abundances derived by Edvardsson et al. (1993; crosses) are shown in the three plots to indicate the trend in metal-rich stars. 
values adopted by Carretta et al. for this star. Using these latter parameters we obtain an LTE oxygen abundance 0.4 dex higher than Carretta et al. (i.e. $\left.[\mathrm{O} / \mathrm{Fe}]_{\mathrm{LTE}}=1.05\right)$, and for a star with these parameters the non-LTE correction to the oxygen abundance is of the order of 0.05 dex (Mishenina et al. 2000), much lower than the 0.25 dex value used by Carretta et al. We therefore arrive at a value $[\mathrm{O} / \mathrm{Fe}]_{\mathrm{NLTE}} \sim 1.0$, in good agreement with the $\mathrm{OH}$ determination by Boesgaard et al. (1999a). Corrections to the stellar parameters as inferred from the non-LTE analysis of Fe lines clearly have an impact on the oxygen abundances which we will address in a forthcoming paper.

Israelian et al. (1998) found four dwarfs in their sample for which oxygen abundances derived using [O I] were in good agreement with those derived from $\mathrm{OH}$ when Hipparcos gravities are used. Several oxygen measurements for unevolved stars based on the [O I] $6300 \AA$ line are compiled in the lower panel of Fig. 1. This figure shows a similar trend than that observed for the abundances recently derived from forbidden lines by Carretta et al. (2000). The presence of a linear trend of $[\mathrm{O} / \mathrm{Fe}]$ versus metallicity in Fig. 1 strongly depends on the only two measurements available at $[\mathrm{Fe} / \mathrm{H}] \leq-2$. These two measurements have been reported by Fulbright \& Kraft (1999) for the subgiants BD $+37^{\circ} 1458$ and BD $+23^{\circ} 3130$, which were also considered by Israelian et al. (1998) and Boesgaard et al. (1999a; only $\mathrm{BD}+37^{\circ} 1458$ in this case). The analysis carried out by Fulbright \& Kraft is based on gravities derived from LTE iron ionization balance of these subgiants where it is well known that non-LTE effects are strong (Thévenin \& Idiart 1999; see also Idiart \& Thévenin, this conference). Allende Prieto et al. (1999) have shown that gravities derived using this technique in metal-poor stars do not agree with the gravities inferred from accurate Hipparcos parallaxes. They find that gravities are systematically underestimated when derived from ionization balances and that upward corrections of $\sim 0.5$ dex can be required at metallicities similar to those of our stars, in good agreement with Thévenin \& Idiart. We remark here that any underestimation of gravities will also strongly underestimate the abundances inferred from the forbidden line. For the two stars under discussion our Hipparcos based gravities are 0.45 and 1.05 dex (for $\mathrm{BD}+37^{\circ} 1458$ and $\mathrm{BD}+23^{\circ} 3130$, respectively) higher than derived by Fulbright \& Kraft, and would imply corrections in the oxygen abundances similar to those indicated in Fig. 1 (a detailed analysis would imply also the correction for the assumed metallicities). Our conclusion is that the uncertainties in the gravities of these subgiants allow the abundances inferred from the forbidden line to be consistent with those estimated from the $\mathrm{OH}$ lines or the triplet. Actually, consistency with the other oxygen indicators is achieved for the high gravities inferred from Hipparcos when consistent analyses are made, and this could be taken as an indication that the high gravities are indeed the correct ones.

\section{Beryllium and oxygen}

The dependence of $\log (\mathrm{Be} / \mathrm{H})$ on $[\mathrm{Fe} / \mathrm{H}]$ and on $[\mathrm{O} / \mathrm{H}]$ (using the abundances derived from the OH UV lines) is essentially linear (García López 1999; Boesgaard et al. 1999b), but with different slopes: $\sim 1.1$ and $\sim 1.5$, respectively. No evidence of a primordial plateau of Be down to $\log (\mathrm{Be} / \mathrm{H})=-13.5$ is found. 


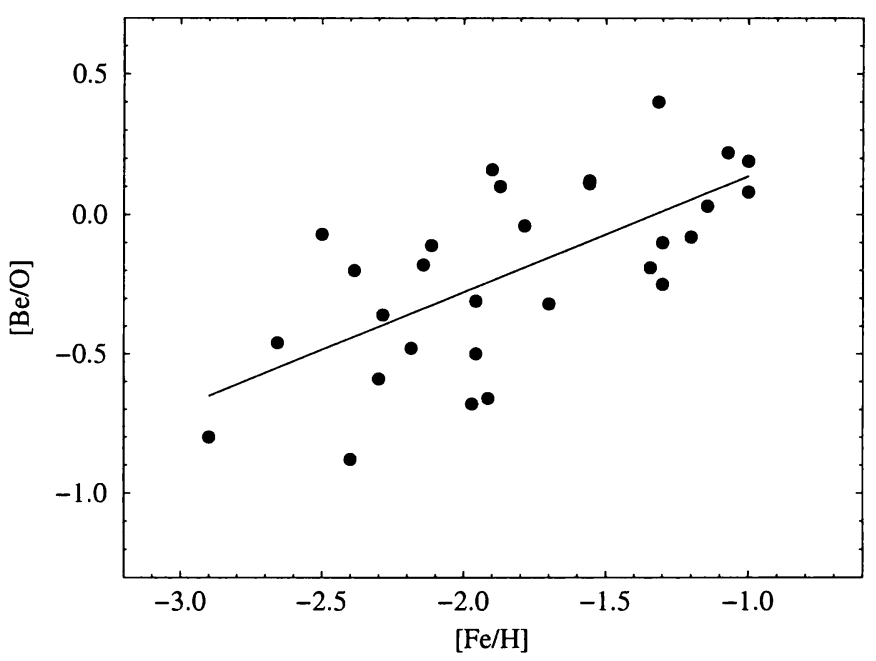

Figure 2. $\quad[\mathrm{Be} / \mathrm{O}]$ vs. $[\mathrm{Fe} / \mathrm{H}]$ for unevolved metal-poor stars. Oxygen abundances used were derived from $\mathrm{OH}$ UV lines. The slope observed in the figure, $0.41 \pm 0.09$, provides an observational constraint to the GCR models.

Figure 2 shows the increase of the $[\mathrm{Be} / \mathrm{O}]$ ratio with increasing metallicity and a slope of $\sim 0.4$. This relation provides an observational constraint to the Galactic Cosmic Ray theories. Three types of GCR models exist at present which try to explain their observed evolution. These are 1) a pure primary GCR from superbubbles (Ramaty, this conference), 2) a hybrid model based on GCR and superbubble accelerated particles (Cassé, this conference), which could be accomplished by a pure superbubble model (Parizot \& Drury, this conference), and 3) standard GCR (Olive, this conference). Apparently all these models can be adopted for both, variable and flat $[\mathrm{O} / \mathrm{Fe}]$. However, models presented by $\mathrm{R}$. Ramaty and $\mathrm{K}$. Olive show more consistency when variable [O/Fe] is adopted.

Chemical evolution models of the early Galaxy where stellar lifetimes are taken into account and assuming that Type Ia SN appear at a Galactic age of 30 million years can also explain the evolution of oxygen delineated in Fig. 1. (Chiappini et al. 1999.). The evolution of oxygen proposed in this paper also helps to understand the evolution of ${ }^{6} \mathrm{Li}$ versus $[\mathrm{Fe} / \mathrm{H}]$ and the ${ }^{6} \mathrm{Li} / \mathrm{Be}$ ratio at low metallicities in the framework of standard Galactic Cosmic Ray Nucleosynthesis (Fields \& Olive 1999). In addition, Ramaty et al. (1999) have proposed that a delay between the effective deposition times into the ISM of Fe and O (only a fraction of which condensed in oxide grains) can explain a linear trend of $[\mathrm{O} / \mathrm{Fe}]$.

It has been suggested (Vangioni-Flam \& Cassé, this conference) to use magnesium as metallicity indicator instead of oxygen. However, given the existence of unevolved halo stars with negative $[\mathrm{Mg} / \mathrm{Fe}]$ ratios (McWilliam 1997; Carney et al. 1997), this approach may not lead to better results. For example, the subdwarf $\mathrm{BD}+3^{\circ} 740$ has $[\mathrm{O} / \mathrm{Fe}] \sim 1$ (see previous Section) while its $[\mathrm{Mg} / \mathrm{Fe}]=-0.28$ (Fuhrmann et al. 1995). Yield of $\mathrm{Mg}$ depends on the extent of mixing (Argast 
et al. 2000) and its primordial abundance can be changed due to the operation of the $\mathrm{MgAl}$ cycle.

\section{References}

Abia, C., \& Rebolo, R. 1989, ApJ, 347, 186

Allende Prieto, C., García López, R. J., Lambert, D. L., \& Gustafsson, B. 1999, ApJ, 527, 879

Allende Prieto, C., \& Lambert, D.L. 2000, AJ, in press

Alonso, A., Arribas, S., \& Martínez-Roger, C. 1996, A\&AS, 313, 873

Argast, D., Samland, M., Gerhard, O. and Thielemann, F. 2000, A\&A, in press Asplund, M., Nordlund, $\AA$, Trampedach, R., \& Stein, R. 1999, A\&A, 346, L17 Balachandran, S., \& Bell, R. 1998, Nature, 392, 791

Barbuy, B. 1988, A\&A, 191, 121

Bessell, M. S., Sutherland, R. S., \& Ruan, K. 1991, ApJ, 383, L71

Boesgaard, A.M., Deliyannis, C. P., King, J.R., Ryan, S.G., Vogt, S.S. \& Beers, T. $1999 \mathrm{~b}, \mathrm{AJ}, 117,1549$

Boesgaard, A.M., King, J.R., Deliyannis, C. P., \& Vogt, S.S. 1999a, AJ, 117, 492

Carney, B., Wright, J., Sneden, C., Laird, J., Aguilar, L. \& Latham, D. 1997, AJ, 114, 363

Carretta, E., Gratton, R. G., \& Sneden, C. 2000, A\&A, in press

Cavallo, R., Pilachowski, C., \& Rebolo, R. 1997, PASP, 109, 226

Chiappini, C., Matteucci, F., Beers, T.C., \& Nomoto, K. 1999, ApJ, 515, 226

Edvardsson, B., Andersen, J., Gustafsson, B., Lambert, D. L., Nissen, P. E., \& Tomkin, J. 1993, A\&A, 275, 101

ESA 1997, The Hipparcos and Tycho Catalogues, ESA SP-1200

Fields, B.D., \& Olive, K.A. 1999, ApJ, 516, 797

Fuhrmann, K., Axer, M., \& Gehren, T. 1995, A\&A, 301, 492

Fulbright, J., \& Kraft, R. 1999, AJ, 118, 527

García López, R.J. 1999, in LiBeB, Cosmic Rays, and Related X- and GammaRays, Eds. R. Ramaty, E. Vangioni-Flam, M. Cassé, \& K. Olive, ASP Conf. Series, 171, p. 77

García López, R.J., Severino, G., \& Gomez, M.T. 1995, A\&A, 297, 787

Israelian, G., García López, R.J., \& Rebolo, R. 1998, ApJ, 507, 805

King, J.R. \& Boesgaard, A.M. 1995, AJ, 109, 383

Kiselman, D. 1993, A\&A, 275, 269

Kraft, R., Sneden, C., Langer, G., \& Prosser, C. 1992, AJ, 104, 645

McWilliam, A. 1997, ARA\&A, 35, 503

Mishenina, T., Korotin, S., Klochkova, V., \& Panchuk, V. 2000, A\&A, 353, 978

Molaro, P. \& Beckman, J. 1984, A\&A, 139, 394

Molaro, P., Beckman, J. \& Castelli, F. 1984, ESA SP-219, 197 
Ramaty, R., Vangioni-Flam, E., Cassé, M., \& Olive, K. 1999, PASP, 111, 651

Rebolo, R., Molaro, P., Abia, C. \& Beckman, J. 1988, A\&A, 193, 193

Spiesman, W., \& Wallerstein, G. 1991, AJ, 102, 1790

Thévenin, F., \& Idiart, T. 1999, ApJ, 521, 753

Tomkin, J., Lemke, M., Lambert, D.L., \& Sneden, C. 1992, AJ, 104, 1568 